\title{
A computer aided classification of certain groups of prime power order: Corrigendum
}

\section{Judith A. Ascione, George Havas, and C.R. Leedham-Green}

The first four paragraphs of $[1, p .258]$ are a mildly erroneous over simplification of the situation. A more accurate description follows.

The analysis of two-generator 3-groups of second maximal class goes along the following lines. We first define a class of group whose structure is particularly amenable to theoretical analysis.

Let $\underline{\underline{P}}$ be a group of order $p^{n}$ and class $m-1$ (for any prime $p$ ) and $s \leq r$ be positive integers such that

(i) $\underline{\underline{P}} / \gamma_{2}(\underline{\underline{P}}) \cong C_{p^{r}} \times C_{p}$ and $\left[\gamma_{i}(\underline{\underline{P}}): \gamma_{i+1}(\underline{P})\right]=p$ for $2 \leq i \leq m-1$, so that $n=m+r-1$.

(ii) Put $\underline{M}_{2}=C_{\underline{\underline{P}}}\left(\gamma_{2}(\underline{\underline{P}}) / \gamma_{4}(\underline{\underline{P}})\right)$. We require $\mathrm{M}_{-2} / \gamma_{2}(\underline{P}) \cong C_{p}{ }_{p-1} \times C_{p}$.

Let $a_{1}$ be a fixed element of $\stackrel{M}{=}$ not lying in the Frattini subgroup of $\underline{\underline{P}}$ with $a_{1}^{p} \in \gamma_{2}(\underline{P})$, and let $\gamma_{1}(\underline{\underline{P}})$ denote $\left\langle\gamma_{2}(\underline{\underline{P}}), a_{1}\right\rangle$.

(iii) For all $i, j \geq 1,\left[\gamma_{i}(\underline{\underline{P}}), \gamma_{j}(\underline{\underline{P}})\right] \subseteq \gamma_{i+j+p^{s-1}}(\underline{\underline{P}})$.

(iv) For all $i \geq 1, \gamma_{i} \underline{\underline{P}}^{p}=\gamma_{i+p}^{s-1}(p-1) \stackrel{(\underline{\underline{p}})}{ }$.

(v) $m \geq p^{s-1}+3$.

Then $\underline{\underline{P}}$ will be said to be a Blackbum group of type $(r, s)$. It

Received 25 August 1977. 
can be shown that conditions (iii) and (iv) are independent of the choice of $a_{1}$ (see [4]).

Here we are concerned with the cases $r=1$ or 2 . If $r=1$, so that $s=1, \stackrel{P}{=}$ is just a $p$-group of maximal class and positive degree of commutativity as defined in [2].

Examples are easily produced. Let 0 denote the ring of integers in the $p^{s}$ th cyclotomic number field, so that 0 is of rank $p^{s-1}(p-1)$ as an abelian group, and let $\theta$ be a primitive $p^{s}$ th root of unity. Let $A$ be the ideal in 0 generated by $\theta-1$, so that $A^{i}$ is of index $p^{i}$ in 0 for all $i>0$. Then the split extension of $0 / A^{m-1}$ by the cyclic group of order $p^{r}$ acting via multiplication by $\theta$ is a Blackburn group of type $(r, s)$ with $O / A^{m-1}$ as a possible choice for $\gamma_{1}(\underline{\underline{P}})$ provided $m \geq p^{s-1}+3$.

The groups of second maximal class with $\underline{P} / \gamma_{2}(\underline{\underline{P}}) \cong C_{9} \times C_{3}$ and of order $3^{n}$, where $n \leq 8$, are analysed in $[1, \S 7]$. Those in $[1$, Table 6] have $\left[\gamma_{i}(\underline{\underline{P}}): \gamma_{i}(\underline{\underline{P}})^{3}\right] \leq 9$ for all $i \geq 2$; such a group we define to be of maximal type. See $[1, \S 4]$ for a general explanation of the tables. All groups descended from group $A$ contain a subgroup of maximal class and index 9 . Those descended from groups $G$ and $H$ are Blackburn groups of type $(2,1)$. The groups in $[1$, Table 7$]$ are of non-maximal type; that is $\left[\gamma_{i}(\underline{\underline{P}}): \gamma_{i}(\underline{\underline{P}})^{3}\right]>9$ if $i \geq 2$ and $\left|\gamma_{i}(\underline{\underline{P}})\right|>9$. This table, when continued indefinitely, will contain all Blackburn 3-groups of type $(2,2)$. It will also contain infinitely many groups with a subgroup of index at most 27 which is of this type, and will contain only a finite number of other groups.

The groups of second maximal class with $\stackrel{P}{=} / \Upsilon_{2} \stackrel{(P)}{=} \cong C_{3} \times C_{3}$ of order $p^{n}$, where $n \leq 10$, are analysed in $[1, \S 6]$. Those in $[1$, Table 2] have $\left[\gamma_{i}(\underline{P}): \gamma_{i}(P)^{3}\right] \leq 9$ for all $i \geq 4$; such groups will also be said to be of maximal type. Those descended from groups $B, O$, and $Q$ contain a subgroup of maximal class and index 9 . Those descended from groups $S$ 
and $U$ contain a Blackburn group of type $(2,1)$ and index 3.

The groups in [1, Tables 4, 5] are descended from groups $H$ and $I$ and are of non-maximal type; that is, $\left[\gamma_{i}(\underline{\underline{P}}): \gamma_{i}(\underline{\underline{P}})^{3}\right]>9$ if $i \geq 4$ and $\left|\gamma_{i}(\underline{P})\right|>9$. It can be shown (see [3]) that all descendents of $H$ and $I$ contain a subgroup $Q$ of index 3 such that $\underline{\underline{Q}}$ has second maximal class, $\underline{Q} / \gamma_{2}(\underline{Q}) \cong C_{9} \times C_{3}$, and $\gamma_{i}(\underline{\underline{Q}})=\gamma_{i+1} \underline{(\underline{P})}$ for all $i \geq 3$. Thus $\underline{\underline{Q}}$ is also of non-maximal type, as in [1, Table 7].

\section{References}

[1] Judith A. Ascione, George Havas, and C.R. Leedham-Green, "A computer aided classification of certain groups of prime power order", Bulz. Austral. Math. Soc. 17 (1977), 257-274; Microfiche supplement, 320 .

[2] N. Blackburn, "On a special class of p-groups", Acta Math. 100 (1958), 45-92.

[3] C.R. Leedham-Green, "Three-groups of second maxir al class", in preparation.

[4] C.R. Leedham-Green, "On p-groups of large class", in preparation.

Department of Mathematics,

Institute of Advanced Studies,

Australian National University,

Canberra, ACT;

Department of Pure Mathematics,

Queen Mary College,

University of London,

London,

England. 